\section{PRESIDENTIAL ADDRESS ON THE}

TREATMENT OF SOME FORIIS OF ALBUIINURIA

Delivered before the Medical Society of London, October 12th, 1896, By REGINALD HARRISON, F.R.C.S.

Kidney Tension and Albuminuria.

EARLY this year, in connection with some observations I published $^{1}$ on kidney tension relative to albuminuria, I narrated the particulars of three cases in which albuminuria of some standing completely and, I believe, permanently disappeared after the digital exploration and puncture or division of the kidney capsule was practised. I will briefly refer again to these cases, together with some others to which my attention has since been called as bearing upon the point to which I desire to direct your attention on this occasion.

It is, however, only right to state that my cases were all instances where the kidney was explored and punctured, or incised, not with the intention of treating an albuminuria but with the object of discovering some other coexisting morbid condition which, though previously suspected, was not on exploration found to exist. I regret that only scanty notes were kept at the time of these and some other cases to which I might have referred, arising from the fact that in the absence of what was more directly sought for, the existing conditions were insufficiently appreciated, whilst the good results accruing appeared at the time either unexplainable, or were referred to local states, such as the accidental fixation of a movable kidney, the division of a disordered nerve, or the moral effects of an operation. It was not until several instances had come under my notice in this way that I began to suspect that a different explanation for the total disappearance of one symptom of disease at least might with some degree of reason be offered. My cases are briefly as follows :

CASE I.- In 1878 I cut down on the kidneyifrom the loin in a youth aged 8, expecting to find a suppuration either within or around the organ. The patient was suspected to have had scarlet fever three weeks before this was done, and had since suffered from intense lumbar pain. He had had a slight rash, some desquamation, a sore throat, and albuminous urine with casts. I undertook the operation with some hesitation, and limited my incision so as just to enable me to put iny finger on the kidney. It felt so tense that I extended my incision and opened it with confidence, expecting to find matter. This was not the case, and I closed the proceeding with the feeling that I had made an error in diagnosis. There was a full discharge of blood and urine from the wound for some days. The latter was lightly plugged with lint, and in the course of ten days or so healed soundly. After the incision was made the excretion of urine became far more abundant, and the albumen gradually and completely disappeared.

CASE II.-In $188_{7}$ I operated upon a man, aged 50 years, who by nature of his occupation .spent a large amount of his time underground. Occasionally he suffered from hæmaturia in conjunction with colicky pains about the groins, and I came to the conclusion that he was suffering from renal calculus. As, however, the symptoms were neither urgent nor confined to one kidney, the consideration of operation.was postponed. In the course of a few months after I first saw him, and whilst he was continuing his work underground, the urine became largely and constantly albuminous, and there was some pain referred to the right loin. I took him into the Royal Infirmary at Liverpool, where I was then residing and explored the right kidney. The organ was found to be enlarged and tense. An incision of an inch in length was made through the cortex, and the pelvis was explored with the finger, but after careful examination no stone could be found. There was a considerable discharge of blood and urine, which continued for a fortnight or so, a drainage tube being retained in the wound; on the withdrawal of the latter healing followed, and the urine became quite normal. I heard some time afterwards that the patient remained in excellent health, and was able to resume his ordinary occupation.

CASE III.--This case is one that came under my observation in 1893 . It was that of a woman, aged 44 , who had suffered from slight hæmaturia a times for a year previously; occasionally the urine wasalbuminous. Shortly after I saw her she had a severe attack of influenza, which was followed by an aggravation of her renal symptoms. She complained of pain pressure over the left kidney, and the albumen not only increased in quantity, but was constantly present in the urine. As she believed she had passed a small calculus some months previously, I thoughtit a proper case for exploration, and this was accordingly made. The late Durham saw the patient in consultation with me. The left kidney was found to be swollen and verv tense. It was opened and explored with th finger, but no calculus could be discovered. There was a free drain of urine, with some blood which continued for about a fortnight, when the wound closed. The patient is now quite well, and the urine normal.

Looking at the three cases I have briefly related, I believe 1 Lancet, January ${ }_{4}$ th, 1896. that the first was scarlatinal nephritis, the second nephritis from exposure to cold and damp, and the last subacute nephritis following most probably upon influenza. Amongst other features, each case was characterised by the presence of albumen in the urine, which I am inclined to attribute to previous inflammation or its immediate effects.

Since these cases were published, Newman, of Glasgow, has recorded two others where albuminuria ceased after the performance of an operation for fixation of the kidney. The cases are thus epitomised ${ }^{2}$ :

CASE A.-Right movable kidney, causing torsion of the ureter and leading to hydronephrosis, albuminuria, and tube casts in the urine. With the onset of paroxysms of pain, hydronephrosis was sometimes present; sometimes it was absent or not observable, but was attended by high specific gravity of the urine and albuminuria, and tube casts always ap peared in the urine at the same time as th

CASE B.-Left movable kidney causing torsion of renal blood vessels, albuminuria, tube casts, severe pain and suppression of urine. There was no hydronephrosis, and operation was succeeded by recovery. The widney was only freely movable, but no increase in size could at any time be made out. Commenting on these cases, Dr. Newman observes: "In the cases of occasional hydronephrosis, the presence of albumen and tube the cases of occasional hydronephrosis, the presence of albumen and tube casts was more difficult to explain. Why did the transient passive hyperæmia lead to the presence of tube casts, the occurrence of which physicians were in the habit of

Both of these cases appear to me to have an important bearing both upon the pathology and treatment of albuminuria. $I$ am indebted to Dr. Hoeber, of Homburg, for the following particulars. He writes : I have seen a very similar case to those you describe, about two months
ago. A man of about 36 years of age, very strong and otherwise perfectly ago. A man of about 36 years of age, very strong and otherwise perfectly healthy, got, after an attack of influenza which did not appear very severe, most intense pain in the right lumbar region associated with slight albuminuria. As the pain lasted over a fortnight, and yielded to no kind of treatment, I sent the patient to a surgeon with the diagnosis of probable renal suppuration, particularly as there was slight feverishness. The incision brought a considerable discharge of blood, but no pus. The at once, and has had no return since.

Surgical Treatment of Kidney Tension.

From a careful consideration of these cases I do not think it is possible to avoid arriving at the conclusion that the disappearance of albumen from the urine was directly connected with the surgical treatment to which in each instance one of the kidneys was submitted. That the organs were in a state of tension, in one class of cases as the result of inflammatory hyperæmia, whilst in the other from 'mechanical vascular obstruction, seems also to be a conclusion which is equally irresistible. Referring to the hyperæmia which exists in the initial stages of nephritis, Sir T. Grainger Stewart observes, "Albuminuria is very often due to changes of an inflammatory character in the tubules and in the stroma of the organ, and in a very large proportion of the cases in which it occurs in practice it is dependent on this cause." That the changes which subsequently ensue as a consequence of nephritis, however commencing, by the substitution of a lower for a higher excretory tissue, as we see in the cirrhosed forms of Bright's disease, is due to the damage the organs received in the early and active stage of inflammation or congestion, seems by no means improbable. Sir Thomas Watson observed in his lectures, "The stress or congestion which befalls the kidney in cases of febrile anasarca may set on foot a morbid process that long works silently and unobserved, but at last declares its operation by symptoms."

Nor is there wanting evidence as to the high state of tension which is sometimes present in the kidney. I have frequently called attention to this at the time of operation for exploration of one of these organs. In one instance the degree would resemble that of a ripe or almost bursting plum, whilst in another the kidney was comparatively flaccid and unresisting on pressure with the finger. Yet these differences were not always at the time explainable.

That the relief of renal tension by direct surgical interference has been proved to be practical and is under certain conditions indicated is also supported by the cases I have cited. Surgeons have long learnt to recognise the disastrous and far-reaching effects of tension as it occurs in the human body whether arising from inflammation or otherwise, and do not hesitate to negative any evil effects it may give rise to by means which render this very unlikely to happen.

2 Clinical Society of London. Lancet, January 18th, 1896. 
Possibly my surgical exir.tien has led me to exaggerate the disastrous effects of tt:asion or tine tiesues of the body, though for the most part it has been gained in regions which may be said to be less highly organised and delicate than the excreting apparatus of the kidney. It certainly seems somewhat remarkable at the present day that so many different views exist, or have been put forward, explanatory of the process by which albumen exudes so as to form a variable part of the urinary excretion. These various theories I shall not attempt to discuss in detail or to reconcile.

ANalogies from the Eye and Testicle.

I may briefly remind you of two analogous conditions. In the eye the most disastrous effects of intra-ocular tension are seen in "glaucoma." The recognition of the true pathology of this affection and the adoption of mechanical treatment by iridectomy or an allied operation for the removal of tension and the prevention of the degenerative changes thus initiated, as first practised by von Graefe, at once resulted in the saving of a large number of eyes which previous to this discovery would undoubtedly have been lost.

In the testicle when it becomes inflamed we have not infrequently transient as well as permanent evidence of the damage that inflammation and tension are capable of bringing about in an organ which, relative to its secreting and investing structures, bears a resemblance to the kidney. The late Mr. Henry Smith was the first to draw attention to certain advantages that followed puncture or limited incision through the capsule of the testicle in acute forms of orchitis. It was alleged, and I believe with a considerable amount of truth, that not only was the pain of tension in this way immediately relieved, but that permanent damage to the secretory structure of the testis was averted. In fact sterility, so far as the organ involved was concerned, was by this means rendered unlikely to occur.

The Structure of the Kidney in relation to Tension.

The kidney may be said to be a highly organised gland surrounded by a thin fibrous capsule, and divided up into compartments or sections by barriers of a similar nature. It is capable of distension to almost any degree by a gradual force acting from within, as, for instance, the retrograde pressure proceeding from strictures which oppose the escape of its excretion, but from the nature of its structural constituents is incapable of adapting itself to sudden emergencies of this kind, as those usually arising in connection with the early stages of acute nephritis.

Assuming, however, that the means I have thus suggested for dealing with intrarenal tension, under circumstances to which I will presently refer, are applicable, it may be urged that as the kidney, as normally disposed, is a double organ, both glands must be directly submitted to the proceedings proposed, inasmuch as in the ordinary form of nephritis both kidneys are usually similarly involved. This, however, does not necessarily follow, as the sympathies existing between the two organs are such as to cause impressions exercised upon one to be reflected on the other. Relief afforded to one kidney, as my cases illustrate, usually assists the other, whilst when the excretory power on one side is suspended or arrested. the opposite organ speedily takes up the whole of this work.

I might illustrate this point further in a variety of ways. The following case, however, seems to me to directly bear upon it.

It was that of a man ayed 30 , who I saw early in 1889 , ten days after he had received an injury to his loins by falling down the hold of a ship and alighting on a case of goods. The right loin was ecchymosed, and from the presence of a little blood in the urine, which continued for some days after the injury, it was concluded that either one or both kidneys had been severely contused. The daily amount of secretion was considerably diminished, and the day I saw him it had only amounted to 16 ounces in the twenty-four hours. There was pain on pressure over the right loin, which remained swollen, discoloured, and tender to the touch, and the temperature had risen and was variable. I thought it probable that perirenal suppuration had occurred. I therefore exposed the right kidney from the loin, and removed some extravasated blood in the course of the incision. The kidney was found very tense and congested. I believed that suppuration had taken place within it, and therefore a small exploring trocar was inserted in two or three places, but without discovering pus. Further, at one point where it was very tense I made an incision pus. the cortex, but only blood and serum escaped. Considering that we had thus got rid of some of the products of the injury which were on the verge of suppuration, the wound was lightly packed with antiseptic lint and left open; there was a free discharge of blood and some urine for some days after, and all the symptoms which rendered the incision desir able, were at once relieved, and the patient made a good and complete recovery.

It was interesting to notice that the excretion of urine was more than doubled in the twenty-four hours following the operation, and the daily amount now remained normal throughout. Here the tendency towards suppression of urine was evidently connected with the intense congestion resulting from the injury in which probably both organs were involved, a condition of the circulation which was at once removed by the treatment described. We do not, I think, sufficiently recognise the high degree of vascular and tubular infarction that attends some grades of nephritis. Some years ago I saw a girl, aged 7, who was suffering from scarlet
fever of a malignant type. Almost complete suppression of urine was the leading feature in the case, and death took place in four days from the leading feature in the case, and death took place in four days rom the commencement of the llness. At the necropsy the kidneys were found
to be so highly congested that $I$ was not surprised at their being unable to be so highly congested that I was not surprised at their being unable
to excrete. I remember the passing impression arising in my mind that to excrete. I remember the passing impression arising in my mind that been effectual in restoring their function.

In a recent paper on Scarlatinal Nephritis and its Varieties, Dr. Meadows Turner" remarks : "Out of the 5,109 cases, 55 died with nephritis, either alone or complicated with other lesions. This number includes those who presented some symptoms during life, as well as some others in whom post mortem extensive disorganisation of the kidney was found, though during life there were no sufficient symptoms for diagnosing such a complication."

\section{Cases in which Reni-puncture may do Good.}

I will now endeavour to indicate the kind of cases of nephritis where it may seem desirable to adopt the practice I have illustrated. In resorting to such measures we cannot entirely, as I have already stated, lose sight of the fact in connection with the subject of albuminuria that its treatment as it at present stands cannot be regarded as entirely satisfactory or progressive. Commenting upon this point, Sir Grainger Stewart, one of our most modern writers on the subject, and whom I had the advantage of conversing with a short time ago in reference to the point that is now before us, observes: "Sir William Roberts and Professor Rosenstein have come to the same general conclusion as Dr. Saundby as regards the inefficacy of drugs in diminishing albuminuria, and I have satisfied myself by a long series of careful observation that we have no right to credit any drug with the power of directly diminishing the discharge of albumen."

The grounds upon which it may be desirable to give relief by surgical means directly applied to the kidney may be illustrated by some of those cases of nephritis which are seen as consequent on scarlet fever, though it seems to me that their application is not necessarily limited to these. In the larger proportion of cases of scarlatinal nephritis the kidney complication is only of a temporary character, and the disappearance of albumen from the urine is both gradual and complete. Under such circumstances surgical interference could not be regarded as warrantable. On the other hand, there are a considerable number of cases met with where this is not so. These may be ranged into two groups. The first includes those instances where the kidney com plication is from the onset of the gravest nature, and death is imminent with more or less suppression of urine, as in the case I have previously referred to, where after death the kidneys were found in a condition of most intense vascular engorgement. In these cases a fatal issue usually ensues most rapidly, the duration of life being largely determined by the degree of suppression that is arrived at.

The second group of cases includes those where after a limited time the tendency, so far as the renal symptoms are principally concerned, is not in the direction of recovery. The amount of albumen does not decrease, tube casts as well as other evidences of disorganisation are found in the urine, and the latter in quantity is below that which may be regarded as an average. Though a physical examination of these organs, either from the loin or by abdominal manipulation, may fail to give any indication as to their condition, tenderness on pressure is often complained of. It is from amongst the cases represented in these two groups that instances will be found where I believe the measures advo-

\footnotetext{
4 Guy's Hospit ll Reports, 1894.
} 11 
cated may sometimes be advantageously practised. It is in these instances that death either rapidly occurs or is brought about no less surely in the course of time by the more chronic forms of nephritis in conjunction with the cardiac complications which so frequently arise in connection with them.

That many cases of nephritis with high tension and subsequent structural deterioration must necessarily be attended with cardiac hypertrophy or enlarged powers of circulation is at once obvious. Diminished capacity to excrete can only be compensated for by increase in the force of the blood current. In the restoration of function we have the only safeguard against the development of this complication.

In conclusion I will offer a few remarks on the precise nature of the surgical treatment of renal tension associated with albuminuria. It is hardly necessary to remind you that by anæsthetics and the antiseptic treatment as developed by Sir Joseph Lister we are now in the position, not only of exploring various organs of the body with perfect safety, but further we are enabled by these means to study what I would speak of as living pathology. It would not be possible for me to illustrate the truth of this observation more vividly than in the case of renal disease. Here by anæsthetics in combination with antiseptics a vast number of diseases have been brought, not only within reach of surgery, but with a degree of success which previously would have been unattainable. The operation of exploring a kidney by an incision from the loin, so as to enable the operator to examine this organ carefully and deliberately with the finger, and, if found necessary, to proceed further, has now been so safeguarded as to remove from the mind of any careful surgeon undertaking it the feeling that he is exposing his patient to any undue risk relative to the reason that is judged to demand it. I cannot say that $I$ ever saw any ill result follow the exposure of the kidney by an incision from the loin for the purpose of its digital exploration. On the other hand, we have seen in numerous directions the necessity for such a proceeding in many cases where, though recovery followed, a correct diagnosis had not previously been arrived at. Such instances include the presence of pus or fluid within the kidney, morbid growths requiring the removal of the organ, stones, undue mobility, and other abnormal conditions.

In the class of cases I am now referring to the kidney should be exposed by a moderate incision from the loin, so as to enable the operator to feel the organ distinctly both in front and behind, aided of course by pressure exercised on the kidney by the hand of an assistant from the front of the abdomen. If in conjunction with the presence of albumen in the urine the kidney is found in a state of tension, such as I have illustrated, three or four punctures may be made through the capsule in various directions; or should the organ be found in a higher state of tension, then a limited incision into the cortex may be practised. After one or other of these measures have been adopted, the wound should be lightly packed with gauze or a drainage tube substituted. In either case the incision should be dressed in such a manner as to provide for the free escape of either blood or urine, or whatever products may be exuded. For this practice I venture to think some reason will be found in the illustrations which have formed the text for my observations this evening.

\section{MEMORANDA:}

MEDICAL, SURGICAL, OBSTETRICAL, THERAPEUTICAL, PATHOLOGICAL, ETc.

\section{CHOREA GRAVIDARUM : INDUCTION OF LABOUR :} DEATH.

K. W., aged 28, primipara, unmarried, came under my care about the middle of July, 1896 . She was then five months pregnant, and had been under the care of another medical man for two months, being treated with iron, iodine, and other remedies, in spite of which the chorea had progressed to an alarming extent. When I first saw her she was standing. She had an idiotic expression on her face, and made peculiar laughing sounds from time to time owing to the in- voluntary contraction of the chest muscles. Her arms and head were thrown about wildly, her hair was matted from her rolling her head about on the pillows when she was lying down; she was unable to drink, as the greater part of the liquid ran out of her mouth; her tongue was much swollen and very dirty ; from the right side of it she had bitten a piece as large as a shilling, and from the other side one the size of a sixpence. She spoke with great difficulty and very indistinctly, as she seemed to have little control over her lips and tongue. No cardiac disease could be detected, but auscultation was difficult owing to the movements being continuous; the heart was not irregular, and the pulse was strong. The involuntary movements continued day and night, so that she never got any sleep. When put into bed her head was dashed about, sometimes with great violence, against the head of the bed; her arms and legs were thrown about in an irregular and violent manner, so that the bedclothes could not be kept on ; from time to time the action of the muscles became so violent that she was thrown over the side of the bed, and unless caught she fell on the floor. When she tried to sit up she did so very awkwardly, and was generally soon thrown back into bed or over the side of it. Any attempt at limiting the movements greatly increased them. Arsenic, bromides, and chloral were tried to relieve the patient, also opium and hypodermics of morphine, but without any relief except for a short period after the latter.

As the patient got no better, and her strength began to fail, after a consultation with my father it was decided to induce abortion. She was put under chloroform, and a bougie was passed between the wall of the uterus and the membranes; then the latter were ruptured so as to reduce tension, hoping possibly in that way to give temporary relief; this relief came, but was only of a few hours' duration, then the involuntary movements came on as severe as ever. Morphine was administered, but with only slight relief. On the third day after rupturing the membranes the fotus was extracted by the leg after chloroform had been given, and the placenta, followed by a profuse hæmorrhage, was removed. The abortion went on satisfactorily, but the patient showed no improvement. On the night following the abortion a hypodermic injection of morphine was given, but without relief, except for a short time. The next day the patient, whose strength had been keeping up well, and who was getting to take more food, suddenly collapsed, sordes rapidly appeared on the lips, her face turned livid, and her extremities went quite cold, and in spite of hot bottles, hypodermics of ether, strychnine, and brandy, she died in about an hour after the change came.

The patient had had "one attack of the dance" when a young girl, and had had rheumatic fever, and belonged to a somewhat neurotic family. She was ill for some time when she had St. Vitus's dance as a girl.

$$
\text { W. E. F. Tinley, M.B., }
$$

Whitbe Senior Obstetric House-Physician, St. Thomas's Hospital.

COMPLETE INVERSION OF THE BLADDER. THis occurred in a female child about 6 months old. The child had an attack of bronchitis some weeks previously, but at the time of the inversion no cause could be assigned for the conditions. The mother first noticed a small red mass gradually protruding through the vulva, which on pressure disappeared. In half an hour the red mass reappeared, but was much larger and could not be returned. When I saw the case (four hours afterwards) it was much congested and about the size of a duck's egg. The urine could be seen at times trickling from the ureters. I at first failed to return it, but by passing a probe-pointed bistoury along the neck of the mass to the urethral orifice, and incising, the inverted bladder was then easily returned. There was no return.

Newcastle-upon-Tyne. Joseph William Leech, M.D.

FIXATION OF THE HEART TO THE DIAPHRAGM. ON making a post-mortem examination the other day I found a condition of things which I think, to say the least, must be uncommon. The subject was a very stout man of 65 , who fell down and expired while taking part in a debate. On examining the heart I found that for a space of about a square 